Pacific

Journal of

Mathematics

\title{
GENERATORS OF THE GAUSS-PICARD MODULAR GROUP
} IN THREE COMPLEX DIMENSIONS

\author{
BaoHua Xie, JieYan Wang And YuePing Jiang
}




\title{
GENERATORS OF THE GAUSS-PICARD MODULAR GROUP IN THREE COMPLEX DIMENSIONS
}

\author{
BaoHua Xie, JieYan Wang And YuePing Jiang
}

\begin{abstract}
In this paper, we will describe a method to obtain the generators system of Gauss-Picard modular group $\mathrm{PU}(3,1 ; \mathbb{Z}[i])$. More precisely, we will show that $\mathrm{PU}(3,1 ; \mathbb{Z}[i])$ can be generated by five given transformations: two Heisenberg translations, two Heisenberg rotations and one involution. Indeed, the same method works for the other higher-dimensional Euclidean Picard modular groups.
\end{abstract}

\section{Introduction}

There are some natural algebraic generalizations of the classical modular group $\operatorname{PSL}(2, \mathbb{Z})$. For example, a Bianchi group is a group of the form $\operatorname{PSL}\left(2, O_{d}\right)$, where $d$ is a positive square-free integer. Here, PSL denotes the projective special linear group and $\mathcal{O}_{d}$ is the ring of integers in the imaginary quadratic number field $\mathbb{Q}(\sqrt{-d})$. These groups were first studied by Bianchi [1892] as a natural class of discrete subgroups of PSL $(2, \mathbb{C})$. A general method to determine finite presentation for each PSL $\left(2, O_{d}\right)$ was developed by Swan [1971] based on the geometrical work of Bianchi, while a separate purely algebraic method was given by Cohn [1968]. As another generalization of the modular group, the construction was generalized by Picard $[1883 ; 1884]$. Suppose H is a Hermitian matrix of signature $(2,1)$ with entries in $\mathrm{O}_{d}$, and let $\mathrm{SU}\left(\mathrm{H} ; \mathrm{O}_{d}\right)$ denote the subgroup of $\mathrm{SU}(\mathrm{H})$ consisting of those matrices whose entries lie in $\mathrm{O}_{d}$. Picard studied the group $\mathrm{PU}\left(\mathrm{H} ; \mathrm{O}_{d}\right)$ acting on the complex hyperbolic plane $\mathbb{U}_{\mathbb{C}}^{2}$. Now, Picard modular groups $\mathrm{PU}\left(\mathrm{H} ; \mathrm{O}_{d}\right)$ have attracted a great deal of attention both for their intrinsic interest as discrete groups and also for their applications in complex hyperbolic geometry.

One can view the modular group or a Bianchi group acting discontinuously on hyperbolic spaces. Then Poincaré's polyhedra theorem provides a geometric method to obtain their generators from their fundamental polyhedra. But [Mostow 1980] told us that the explicit construction of fundamental domains for lattices in complex hyperbolic spaces was particularly difficult. Until recently, the geometry of SU(H; $\left.\mathrm{O}_{3}\right)$ had been studied by Falbel and Parker [2006], while the geometry of

MSC2010: primary 32M05, 22E40; secondary 32M15.

Keywords: complex hyperbolic space, Picard modular groups, generators. 
SU(H; $\left.\mathrm{O}_{1}\right)$ had been studied by Francsics and Lax [2005a; 2005b; 2006] and Falbel, Francsics and Parker [Falbel et al. 2011b]. By applying similar ideas to those of [Falbel and Parker 2006; Falbel et al. 2011b], Zhao [2012] obtained generators of the Euclidean Picard groups PU $\left(2,1 ; \mathrm{O}_{d}\right)$ for $d=2,7,11$.

There are some simple algorithms to obtain the generators of the modular group or some Picard modular groups. For example, the continued fraction algorithm may be applied to any element of the modular group $\operatorname{PSL}(2, \mathbb{Z})$. This shows that $S(z)=-1 / z$ and $T(z)=z+1$ generate $\operatorname{PSL}(2, \mathbb{Z})$. This algorithm was extended to $\mathrm{PU}\left(2,1 ; \mathfrak{O}_{1}\right)$ in [Falbel et al. 2011a], which provided a different system of generators from those obtained via a fundamental domain in [Falbel et al. 2011b]. In [Wang et al. 2011], the authors applied the continued fraction algorithm to $\mathrm{PU}\left(2,1 ; \mathrm{O}_{3}\right)$ and produced a different system of generators from that obtained in [Falbel and Parker 2006].

There is an obvious generalization of Picard modular groups to higher complex dimensions. We observe that very little is known about the geometry and algebraic properties, e.g., explicit fundamental domain or generating system of the higherdimensional Picard modular groups $\mathrm{PU}\left(n, 1 ; \mathrm{O}_{d}\right)$. In [Xie et al. 2013], the continued fraction algorithm was generalized to Picard modular groups in higher complex dimensions. It contained the first generalization that we were aware of to a group of $4 \times 4$ matrices. However, it seems very difficult to extend the continued fraction algorithm to other higher-dimensional Picard modular groups. Using a combination of the ideas from [Falbel et al. 2011a; Xie et al. 2013] and [Falbel and Parker 2006; Zhao 2012], we will present a method to obtain the generating system of the Gauss-Picard modular group PU( 3,$1 ; \mathbb{Z}[i])$. We first get the generators of the stabilizer of infinity of $\mathrm{PU}(3,1 ; \mathbb{Z}[i])$ by applying a similar argument as in our previous paper [Xie et al. 2013]. Then we will construct a subset in the boundary of complex hyperbolic space which contains the fundamental domain for the stabilizers of infinity in $\mathrm{PU}(3,1 ; \mathbb{Z}[i])$. Finally, we will show the boundaries of some isometric spheres that contain this subset. This method works for the other higher-dimensional Euclidean Picard modular groups.

\section{Preliminaries}

2.1. The Siegel domain. We recall some basic notions of complex hyperbolic geometry. For more details we refer the reader to [Goldman 1999; Parker 2010].

Let $\mathbb{C}^{n, 1}$ denote the vector space $\mathbb{C}^{n+1}$ equipped with the Hermitian form of signature $(n, 1)$ given by the matrix

$$
J=\left(\begin{array}{ccc}
0 & 0 & 1 \\
0 & I_{n-1} & 0 \\
1 & 0 & 0
\end{array}\right) .
$$


The Hermitian product of two vectors $z$ and $\boldsymbol{w}$ is given by $\langle\boldsymbol{z}, \boldsymbol{w}\rangle=\boldsymbol{w}^{*} J \boldsymbol{z}$, where $\boldsymbol{w}^{*}$ denotes the Hermitian transpose of $\boldsymbol{w}$.

We denote by $V_{-}$and $V_{0}$ the negative and null cones associated to the Hermitian form, respectively. The complex hyperbolic $n$-space $\mathbb{U}_{\mathbb{C}}^{n}$ is the projectivization of $V_{-}$, and its boundary is the projectivization of $V_{0}$. The model of $\mathbb{U}_{\mathbb{C}}^{n}$ associated to the Hermitian form given above is often referred to as the Siegel model of $\mathbb{Q}_{\mathbb{C}}^{n}$.

We define the Siegel domain $\mathfrak{S}$ of the complex hyperbolic $n$-space $\mathbb{U}_{\mathbb{C}}^{n}$ by identifying points of $\mathfrak{S}$ with their horospherical coordinates,

$$
z=(\zeta, v, u) \in \mathbb{C}^{n-1} \times \mathbb{R} \times \mathbb{R}^{+} .
$$

The boundary of $\mathfrak{S}$ is given by $H_{0} \cup\left\{q_{\infty}\right\}$, where $q_{\infty}$ is a distinguished point at infinity and $H_{0}=\mathbb{C}^{n-1} \times \mathbb{R} \times\{0\}$.

2.2. Heisenberg group. The boundary of a complex hyperbolic space is identified with the one-point compactification of the Heisenberg group. The $(2 n-1)$ dimensional Heisenberg group $\mathscr{H}_{2 n-1}$ is $\mathbb{C}^{n-1} \times \mathbb{R}$ with the group law

$$
(\xi, v) \cdot(z, u)=(\xi+z, v+u+2 \mathfrak{s}\langle\langle\xi, z\rangle) .
$$

Here $\langle\langle\xi, z\rangle\rangle=z^{*} \xi$ is the standard positive definite Hermitian form on $\mathbb{C}^{n-1}$. In particular, we write $\|\xi\|^{2}=\xi^{*} \xi$.

The Heisenberg group acts on itself by Heisenberg translation. For $(\tau, t) \in \mathscr{H}_{2 n-1}$, this translation is

$$
N_{(\tau, t)}(\xi, v)=(\tau+\xi, t+v+2 \mathfrak{s}\langle\langle\tau, \xi\rangle) .
$$

The unitary group $U(n-1)$ acts on the Heisenberg group by Heisenberg rotation.

2.3. Holomorphic isometries. Define a map $\mathfrak{S} \rightarrow \mathbb{C} \mathbb{P}^{n}$ by

$$
\psi:(\xi, \nu, u) \mapsto\left(\begin{array}{c}
\frac{1}{2}\left(-\|\xi\|^{2}-u+i v\right) \\
\xi \\
1
\end{array}\right), \quad \psi: q_{\infty} \mapsto\left(\begin{array}{c}
1 \\
0 \\
\vdots \\
0
\end{array}\right) .
$$

Then $\psi$ maps the set of points $z \in \mathfrak{S}$ homeomorphically to the set of points $z \in \mathbb{C} \mathbb{P}^{n}$ with $\langle z, z\rangle<0$, and maps the set of points in $\partial \mathfrak{S}$ homeomorphically to the set of points $z \in \mathbb{C P}^{n}$ with $\langle z, z\rangle=0$. We write $\psi(z)=z$.

The Bergman metric on $\mathfrak{S}$ is given by the distance formula

$$
\cosh ^{2} \frac{\rho(z, w)}{2}=\frac{\langle\boldsymbol{z}, \boldsymbol{w}\rangle\langle\boldsymbol{w}, \boldsymbol{z}\rangle}{\langle\boldsymbol{z}, \boldsymbol{z}\rangle\langle\boldsymbol{w}, \boldsymbol{w}\rangle} .
$$

The holomorphic isometry group of $\mathfrak{S}$ with respect to the Bergman metric is the projective unitary group $\mathrm{PU}(n, 1)$, and it acts on $\mathbb{C} \mathbb{P}^{n}$ by matrix multiplication. 
2.4. Picard modular groups. Let $O_{d}$ be the ring of integers in the imaginary quadratic number field $\mathbb{Q}(i \sqrt{d})$, where $d$ is a positive square-free integer. If $d \equiv 1,2 \bmod 4$, then $O_{d}=\mathbb{Z}[\sqrt{d} i]$, and if $d \equiv 3 \bmod 4$, then $O_{d}=\mathbb{Z}[(1+\sqrt{d} i) / 2]$. The subgroup of $\mathrm{PU}(n, 1)$ with entries in $\mathrm{O}_{d}$ is called the Picard modular group for $O_{d}$ and is written as $\mathrm{PU}\left(n, 1 ; \mathrm{O}_{d}\right)$. Obviously, if $d=1$, then the ring $\mathrm{O}_{d}$ can be written as $\mathbb{Z}[i]$.

Remark 1. The matrices corresponding to the generators obtained in this paper belong to the group $U(3,1 ; \mathbb{Z}[i])$. In relation to complex hyperbolic isometries, the relevant group is $\mathrm{PU}(3,1 ; \mathbb{Z}[i])=\mathrm{SU}(3,1 ; \mathbb{Z}[i]) / \mathbb{Z}_{4}$. The center of $\mathrm{SU}(3,1)$ is isomorphic to $\mathbb{Z}_{4}$, the group of fourth roots of unity. By abuse of notation, we will denote the Gauss-Picard modular group in three complex dimensions by $U(3,1 ; \mathbb{Z}[i])$.

2.5. Heisenberg automorphism groups. The action of Heisenberg isometries extends to the Siegel domain, fixing $q_{\infty}$. Some examples of Heisenberg isometries are as follows: for $U \in U(n-1)$ and $(\tau, t) \in \mathscr{H}_{2 n-1}$, the Heisenberg rotation and Heisenberg translation correspond to the matrices

$$
M_{U}=\left(\begin{array}{lll}
1 & 0 & 0 \\
0 & U & 0 \\
0 & 0 & 1
\end{array}\right) \quad \text { and } \quad N_{(\tau, t)}=\left(\begin{array}{ccc}
1 & -\tau^{*} & \frac{1}{2}\left(-\|\tau\|^{2}+i t\right) \\
0 & I_{n-1} & \tau \\
0 & 0 & 1
\end{array}\right)
$$

in $\mathrm{SU}(n, 1)$, respectively. The Heisenberg dilation by $r$ fixing $q_{\infty}$ and 0 corresponds to the matrix $A_{r} \in \mathrm{SU}(n, 1)$, where

$$
A_{r}=\left(\begin{array}{ccc}
r & 0 & 0 \\
0 & I_{n-1} & 0 \\
0 & 0 & 1 / r
\end{array}\right)
$$

Finally, the Heisenberg inversion interchanging $q_{\infty}$ and 0 corresponds to the matrix $R \in \mathrm{SU}(n, 1)$, where

$$
R=\left(\begin{array}{ccc}
0 & 0 & 1 \\
0 & -I_{n-1} & 0 \\
1 & 0 & 0
\end{array}\right)
$$

2.6. Isometric spheres. Given an element $G \in \mathrm{PU}(3,1)$ such that $G\left(q_{\infty}\right) \neq q_{\infty}$, we define the isometric sphere of $G$ to be the hypersurface

$$
\left\{z \in \mathbb{U}_{\mathbb{C}}^{3}:\left|\left\langle z, q_{\infty}\right\rangle\right|=\left|\left\langle z, G^{-1}\left(q_{\infty}\right)\right\rangle\right|\right\} .
$$


For example, the isometric sphere of

$$
R=\left(\begin{array}{cccc}
0 & 0 & 0 & 1 \\
0 & -1 & 0 & 0 \\
0 & 0 & -1 & 0 \\
1 & 0 & 0 & 0
\end{array}\right)
$$

is

$$
\mathscr{B}_{0}=\left\{\left(\zeta_{1}, \zeta_{2}, t, u\right) \in \mathfrak{S}:\left.|| \zeta_{1}\right|^{2}+\left|\zeta_{2}\right|^{2}+u+i t \mid=2\right\}
$$

in horospherical coordinates.

All other isometric spheres are images of $\mathscr{B}_{0}$ by Heisenberg dilations, rotations and translations. Thus, the isometric sphere with radius $r$ and center $\left(\zeta_{1}^{0}, \zeta_{2}^{0}, t^{0}, 0\right)$ is given by

$$
\left\{\left(\zeta_{1}, \zeta_{2}, t, u\right):|| \zeta_{1}-\left.\zeta_{1}^{0}\right|^{2}+\left|\zeta_{2}-\zeta_{2}^{0}\right|^{2}+u+i t-i t^{0}+2 i \Im\left(\zeta_{1} \overline{\zeta_{1}^{0}}+\zeta_{2} \bar{\zeta}_{2}^{0}\right) \mid=r^{2}\right\} .
$$

If $G$ has the matrix form

$$
\left(\begin{array}{llll}
a_{11} & a_{12} & a_{13} & a_{14} \\
a_{21} & a_{22} & a_{23} & a_{24} \\
a_{31} & a_{32} & a_{33} & a_{34} \\
a_{41} & a_{42} & a_{43} & a_{44}
\end{array}\right),
$$

then $G\left(q_{\infty}\right) \neq q_{\infty}$ if and only if $a_{41} \neq 0$. The isometric sphere of $G$ has radius $r=\sqrt{2 /\left|a_{41}\right|}$ and center $G^{-1}\left(q_{\infty}\right)$, which in horospherical coordinates is

$$
\left(\zeta_{1}^{0}, \zeta_{2}^{0}, t^{0}, 0\right)=\left(\overline{a_{42}} / \overline{a_{41}}, \overline{a_{43}} / \overline{a_{41}}, 2 \Im\left(\overline{a_{44}} / \overline{a_{41}}\right), 0\right) .
$$

\section{The generators of the stabilizer}

Let $\Gamma_{\infty}$ be the stabilizer subgroup of $q_{\infty}$ in $\mathrm{PU}(n, 1)$. That is,

$$
\Gamma_{\infty} \equiv\left\{g \in \mathrm{PU}(n, 1): g\left(q_{\infty}\right)=q_{\infty}\right\} .
$$

We recall from [Falbel et al. 2011a; Francsics and Lax 2005a; 2005b; Xie et al. 2013] that the Langlands decomposition can be used to parametrize a transformation in the stabilizer subgroup of $q_{\infty}$.

Lemma 2 (Langlands decomposition). Any element $P \in \Gamma_{\infty}$ can be decomposed as a product of a Heisenberg translation, dilation, and a rotation:

$$
P=N_{(\tau, t)} A_{r} M_{U}=\left(\begin{array}{ccc}
r & -\tau^{*} U & \left(-\|\tau\|^{2}+i t\right) / 2 r \\
0 & U & \tau / r \\
0 & 0 & 1 / r
\end{array}\right) .
$$

The parameters satisfy the corresponding conditions. That is, $U \in U(n-1), r \in \mathbb{R}^{+}$ and $(\tau, t) \in \mathscr{H}_{2 n-1}$. 
First, we describe the Heisenberg rotations in the Gauss-Picard modular group $U(3,1 ; \mathbb{Z}[i])$. Let $U(2 ; \mathbb{Z}[i])$ be the unitary group $U(2)$ over the ring $\mathbb{Z}[i]$. Then we have the following result.

Lemma 3. $U(2 ; \mathbb{Z}[i])$ can be generated by the two unitary matrices

$$
U_{1}=\left(\begin{array}{ll}
0 & 1 \\
1 & 0
\end{array}\right) \text { and } U_{2}=\left(\begin{array}{ll}
i & 0 \\
0 & 1
\end{array}\right)
$$

Remark 4. A similar lemma was proved for $U(2 ; \mathbb{Z}[(1+\sqrt{-3}) / 2])$ in $[\mathrm{Xie}$ et al. 2013].

Next, we characterize the elements of the stabilizer subgroup $\Gamma_{\infty}$ of infinity in the Picard modular group $U(3,1 ; \mathbb{Z}[i])$. We denote this stabilizer by $\Gamma_{\infty}(3,1 ; \mathbb{Z}[i])$.

Lemma 5. An element $P \in U(3,1 ; \mathbb{Z}[i])$ lies in $\Gamma_{\infty}(3,1 ; \mathbb{Z}[i])$ if and only if the parameters in the Langlands decomposition of $P$ satisfy the conditions

$$
r=1, \quad t \in 2 \mathbb{Z}, \quad \tau=\left(\tau_{1}, \tau_{2}\right)^{T} \in \mathbb{Z}[i]^{2}, \quad U \in U(2 ; \mathbb{Z}[i]), \quad\|\tau\| \in 2 \mathbb{Z} .
$$

Proof. The proof of this lemma follows from the Langlands decomposition form of $P \in \Gamma_{\infty}(3,1 ; \mathbb{Z}[i])$.

We are now in a position to determine the generators of the stabilizer subgroup of $q_{\infty}$.

Proposition 6. Let $\Gamma_{\infty}(3,1 ; \mathbb{Z}[i])$ be stated as above. Then $\Gamma_{\infty}(3,1 ; \mathbb{Z}[i])$ is generated by the Heisenberg translations $N_{\left((1,1)^{T}, 0\right)}, N_{\left((0,0)^{T}, 2\right)}$ and the Heisenberg rotations $M_{U_{i}}(i=1,2)$.

Proof. Our proof starts with the observation that there is no dilation component of $P \in \Gamma_{\infty}(3,1 ; \mathbb{Z}[i])$ in its Langlands decomposition. That is, $P$ must have the form

$$
P=N_{(\tau, t)} M_{U}=\left(\begin{array}{ccc}
1 & -\tau^{*} & \left(-\|\tau\|^{2}+i t\right) / 2 \\
0 & I_{2} & \tau \\
0 & 0 & 1
\end{array}\right)\left(\begin{array}{ccc}
1 & 0 & 0 \\
0 & U & 0 \\
0 & 0 & 1
\end{array}\right)
$$

Since the unitary matrix $U$ lies in $U(2 ; \mathbb{Z}[i])$, the rotation component of $P$ in the Langlands decomposition is generated by $M_{U_{i}}(i=1,2)$ by Lemma 3 .

What is left is to consider the Heisenberg translation part $N_{(\tau, t)}$ of $P$. Let

$$
\tau=\left(m_{1}+n_{1} i, m_{2}+n_{2} i\right)^{T},
$$

where $m_{1}, n_{1}, m_{2}, n_{2} \in \mathbb{Z}$. Since $|\tau|^{2}=m_{1}^{2}+n_{1}^{2}+m_{2}^{2}+n_{2}^{2} \in 2 \mathbb{Z}$, there are two cases:

(1) $m_{1}^{2}+n_{1}^{2} \in 2 \mathbb{Z}$ and $m_{2}^{2}+n_{2}^{2} \in 2 \mathbb{Z}$;

(2) $m_{1}^{2}+n_{1}^{2} \in 2 \mathbb{Z}+1$ and $m_{2}^{2}+n_{2}^{2} \in 2 \mathbb{Z}+1$. 
We first consider the case (1). We can write $\tau$ as

$$
\tau=\left(k_{1}(1+i)+l_{1}(1-i), k_{2}(1+i)+l_{2}(1-i)\right),
$$

where $k_{1}, l_{1}, k_{2}, l_{2} \in \mathbb{Z}$. $N_{(\tau, t)}$ splits as

$$
N_{(\tau, t)}=N_{\left((0,0)^{T}, t\right)} \circ N_{(\tau, 0)} .
$$

Since $t=2 k \in 2 \mathbb{Z}, N_{\left((0,0)^{T}, t\right)}=N_{\left((0,0)^{T}, 2\right)}^{k}$. We also have

$$
N_{(\tau, 0)}=N_{\left((1+i, 0)^{T}, 0\right)}^{k_{1}} \circ N_{\left((i-1,0)^{T}, 0\right)}^{l_{1}} \circ N_{\left((0,0)^{T}, 2\right)}^{2 k_{1} l_{1}} \circ N_{\left((0,1+i)^{T}, 0\right)}^{k_{2}} \circ N_{\left((0,1+i)^{T}, 0\right)}^{l_{2}} \circ N_{\left((0,0)^{T}, 2\right)}^{-2 k_{2} l_{2}} .
$$

We observe that

$$
\begin{aligned}
& N_{\left((1+i, 0)^{T}, 0\right)}=N_{\left((1,1)^{T}, 0\right)} \circ N_{\left((i,-1)^{T}, 0\right)} \circ N_{\left((0,0)^{T}, 2\right)}, \\
& N_{\left((i-1,0)^{T}, 0\right)}=N_{\left((i, 1)^{T}, 0\right)} \circ N_{\left((1,1)^{T}, 0\right)}^{-1} \circ N_{\left((0,0)^{T}, 2\right)}^{-1}, \\
& N_{\left((0,1+i)^{T}, 0\right)}=N_{\left((1,1)^{T}, 0\right)} \circ N_{\left((-1, i)^{T}, 0\right)} \circ N_{\left((0,0)^{T}, 2\right),}, \\
& N_{\left((0, i-1)^{T}, 0\right)}=N_{\left((1, i)^{T}, 0\right)} \circ N_{\left((1,1)^{T}, 0\right)}^{-1} \circ N_{\left((0,0)^{T}, 2\right)}^{-1} .
\end{aligned}
$$

It is easy to see that

$$
\begin{aligned}
N_{\left((i, 1)^{T}, 0\right)} & =M_{U_{2}} N_{\left((1,1)^{T}, 0\right)} M_{U_{2}}^{-1}, \\
N_{\left((i,-1)^{T}, 0\right)} & =M_{U_{1}} M_{U_{2}}^{2} M_{U_{1}} M_{U_{2}} N_{\left((1,1)^{T}, 0\right)} M_{U_{2}}^{3}\left(M_{U_{1}} M_{U_{2}} M_{U_{1}}\right)^{2}, \\
N_{\left((-1, i)^{T}, 0\right)} & =M_{U_{2}}^{2} M_{U_{1}} M_{U_{2}} M_{U_{1}} N_{\left((1,1)^{T}, 0\right)} M_{U_{2}}^{2}\left(M_{U_{1}} M_{U_{2}} M_{U_{1}}\right)^{3}, \\
N_{\left((1, i)^{T}, 0\right)} & =M_{U_{1}} M_{U_{2}} M_{U_{1}} N_{\left((1,1)^{T}, 0\right)}\left(M_{U_{1}} M_{U_{2}} M_{U_{1}}\right)^{3} .
\end{aligned}
$$

In case (2), similar considerations apply to the translation $N_{(\tau, 0)} \circ N_{\left((1,1)^{T}, 0\right)}$, where $N_{(\tau, 0)}$ belongs to case (1).

\section{Fundamental domain for the stabilizer in $\operatorname{PU}(2,1 ; \mathbb{Z}[i])$}

In [Falbel et al. 2011b], the authors described a method to find the fundamental domain for the stabilizer of $q_{\infty}$ in the Gauss-Picard modular group PU(2, $\left.1 ; \mathbb{Z}[i]\right)$ in two complex dimensions. We review it now.

Let $\Gamma$ be PU $(2,1 ; \mathbb{Z}[i])$ and $\Gamma_{\infty}$ be the stabilizer of $q_{\infty}$. Every element of $\Gamma_{\infty}$ is upper triangular, and its diagonal entries are units in $\mathbb{Z}[i]$. Recall that the units of $\mathbb{Z}[i]$ are $\pm 1, \pm i$. Therefore $\Gamma_{\infty}$ contains no dilations and fits into the exact sequence

$$
0 \longrightarrow \mathbb{Z} \longrightarrow \Gamma_{\infty} \stackrel{\Pi_{*}}{\longrightarrow} \Delta \longrightarrow 1,
$$

where $\Delta \subset$ Isom $\mathbb{Z}[i]$ is of index 2 and $\Pi$ is the vertical projection defined by $\Pi:(z, t) \in \mathscr{H} \mapsto z \in \mathbb{C}$. 


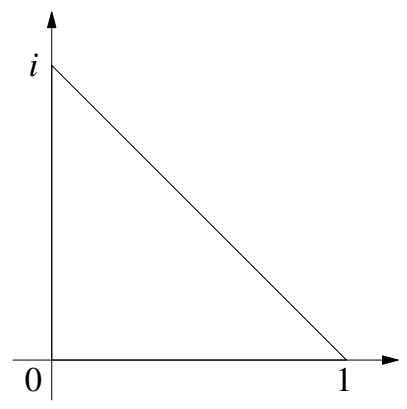

Figure 1. A fundamental domain for the index two subgroup $\Delta \subset$ Isom $\mathbb{Z}[i]$ is a triangle $\Delta$ with vertices $0,1, i$.

As the first step toward the construction of a fundamental domain for the action of $\Gamma_{\infty}$ on $\mathscr{H}$, one should construct a fundamental domain in $\mathbb{C}$ of $\Delta \subseteq$ Isom $\mathbb{Z}[i]$. From the generators of $\Delta$, one finds that a fundamental domain for $\Delta \subseteq \operatorname{Isom} \mathbb{Z}[i]$ is the triangle $\Delta$ with vertices $0,1, i$; see Figure 1 .

In order to produce a fundamental domain for $\Gamma_{\infty}$, we look at all the preimages of the triangle (that is, a fundamental domain of $\Pi_{*}\left(\Gamma_{\infty}\right)$ ) under the vertical projection $\Pi$ and we intersect this with a fundamental domain for $\operatorname{ker}\left(\Pi_{*}\right)$. The inverse image of the triangle under $\Pi$ is an infinite prism. The kernel of $\Pi_{*}$ is the infinite cyclic group generated by $T$, the vertical translation by $(0,2)$. Hence, a fundamental domain for $\Gamma_{\infty}$ is the prism in $\mathscr{H}$ with vertices $(0, \pm 1),(1, \pm 1),(i, \pm 1)$.

\section{Statement of the results}

In this section, we recall the geometric method used in [Falbel and Parker 2006; Falbel et al. 2011b] to determine the generators of the Euclidean Picard groups, and then state our method and results.

The geometric method is based on the special feature that the Euclidean Picard modular orbifold has only one cusp for $d=1,2,3,7,11$. The basic idea of the proof can be described easily. Analogously to Theorem 3.5 of [Falbel and Parker 2006], it can be proved that $\left\langle\Gamma_{\infty}, R\right\rangle$ has only one cusp. The fact that $\mathrm{PU}\left(2,1 ; \mathrm{O}_{d}\right)$ has the same cusp and the stabilizer of infinity as the group generated by $\left\langle\Gamma_{\infty}, R\right\rangle$ shows that they are the same. The key step is to find a union of isometric spheres so that a fundamental domain for $\Gamma_{\infty}$ is contained in the intersection of their exteriors and a fundamental domain for the stabilizer, which implies that the group $\left\langle\Gamma_{\infty}, R\right\rangle$ has only one cusp. In other words, one should show that the union of the boundaries of these isometric spheres in the Heisenberg group contains a fundamental domain for the stabilizer of infinity.

We will prove our result by using a similar idea. The main observation is that there is no need to know the exact fundamental domain for the stabilizer of infinity. 
We will construct a set in the Heisenberg group which contains a fundamental set for the stabilizer of infinity as a subset. Then we show that the union of the boundaries of some isometric spheres in the Heisenberg group covers this set. This also show that the group $\left\langle\Gamma_{\infty}, R\right\rangle$ has only one cusp.

More precisely, let $\Sigma$ be the set

$$
\left\{\left(\xi_{1}, \xi_{2}, t\right): \xi_{i} \in \Delta,-1 \leq t \leq 1\right\} .
$$

Here $\Delta$ is the fundamental domain of $\Delta \subset$ Isom $\mathbb{Z}[i]$.

Note that $\Sigma$ is not a fundamental domain for the stabilizer of infinity because this set is preserved by some Heisenberg rotations.

Proposition 7. $\Sigma$ contains a fundamental domain for the stabilizer of infinity.

Proof. The restriction of the action of the stabilizer of infinity on each copy of $\mathbb{C}$ has the same fundamental domain $\Delta$ as $\Delta \subset$ Isom $\mathbb{Z}[i]$. Then $\Sigma$ is the preimage of $\Delta x \Delta$ under vertical projection intersected with a fundamental domain for the vertical translation by $\left((0,0)^{T}, 2\right)$. It is clear that $\Sigma$ is preserved by the Heisenberg rotations $M_{U_{1}}$. Hence, a fundamental domain for $\Gamma_{\infty}$ lies inside $\Sigma$.

In next section we will prove our main theorem. Our main step is to show that $\Sigma$ lies inside the boundaries of some isometric spheres in the Heisenberg group. It is obvious that the geodesic cone from $q_{\infty}$ over $\Sigma$ contains a fundamental domain for the Gauss-Picard modular group $U(3,1 ; \mathbb{Z}[i])$.

Theorem 8. The Picard modular group $U(3,1 ; \mathbb{Z}[i])$ is generated by the Heisenberg translations

$$
N_{\left((1,1)^{T}, 0\right)}=\left(\begin{array}{rrrr}
1 & -1 & -1 & -1 \\
0 & 1 & 0 & 1 \\
0 & 0 & 1 & 1 \\
0 & 0 & 0 & 1
\end{array}\right), \quad N_{\left((0,0)^{T}, 2\right)}=\left(\begin{array}{llll}
1 & 0 & 0 & i \\
0 & 1 & 0 & 0 \\
0 & 0 & 1 & 0 \\
0 & 0 & 0 & 1
\end{array}\right),
$$

the Heisenberg rotations

$$
M_{U_{1}}=\left(\begin{array}{cccc}
1 & 0 & 0 & 0 \\
0 & 0 & 1 & 0 \\
0 & 1 & 0 & 0 \\
0 & 0 & 0 & 1
\end{array}\right), \quad M_{U_{2}}=\left(\begin{array}{llll}
1 & 0 & 0 & 0 \\
0 & i & 0 & 0 \\
0 & 0 & 1 & 0 \\
0 & 0 & 0 & 1
\end{array}\right),
$$

and the involution

$$
R=\left(\begin{array}{rrrr}
0 & 0 & 0 & 1 \\
0 & -1 & 0 & 0 \\
0 & 0 & -1 & 0 \\
1 & 0 & 0 & 0
\end{array}\right)
$$




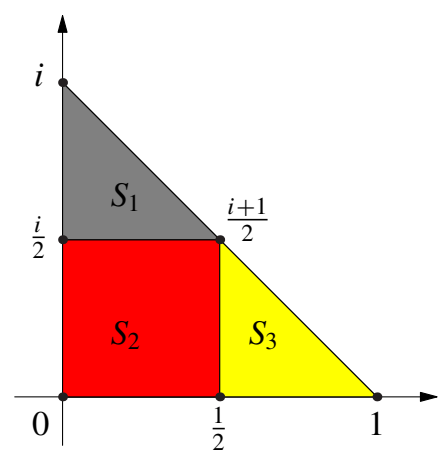

Figure 2. The decomposition of the fundamental domain $\triangle$ for $\Delta \subset$ Isom $\mathbb{Z}[i]$ into three parts.

\section{Proof of Theorem 8}

In this section we will prove that the generators of Picard modular groups consist of the generators of the stabilizer and the involution.

Recall that the Cygan sphere $\mathscr{B}_{0}$ is the isometric sphere of $R$. The boundary $\mathscr{S}_{0}$ of $\mathscr{S}_{0}$ is called the Heisenberg sphere in the Heisenberg group. $\mathscr{Y}_{0}$ is defined by

$$
\mathscr{S}_{0}=\left\{\left.|| \xi_{1}\right|^{2}+\left|\xi_{2}\right|^{2}+t i \mid=2\right\} .
$$

Indeed, we only need to consider the boundaries of isometric spheres in the Heisenberg group because two isometric spheres have a nonempty interior intersection if and only if the boundaries have a nonempty interior intersection.

It is not hard to see that parts of $\Sigma$ lie outside $\mathscr{S}_{0}$. Therefore we need to find more isometric spheres whose boundaries together with $\mathscr{S}_{0}$ contain the set $\Sigma$.

Note that $\Sigma$ has the form

$$
\Sigma=\left\{\left(\xi_{1}, \xi_{2}, t\right): \xi_{1} \in \Delta, \xi_{2} \in \Delta,-1 \leq t \leq 1\right\} .
$$

First, we decompose $\triangle$ into three parts. We write $\Delta=S_{1} \cup S_{2} \cup S_{3}$, where $S_{1}$ is a triangle with vertices $i, \frac{i}{2}, \frac{1}{2}(1+i), S_{2}$ is a square with vertices $0, \frac{i}{2}, \frac{1}{2}, \frac{1}{2}(1+i)$, and $S_{3}$ is a triangle with vertices $0,1, \frac{1}{2}(1+i)$; see Figure 2 .

Therefore, $\Sigma$ will be decomposed into nine subsets:

- $\Sigma_{1}=\left\{\left(\xi_{1}, \xi_{2}, t\right): \xi_{1} \in S_{1}, \xi_{2} \in S_{1},-1 \leq t \leq 1\right\}$,

- $\Sigma_{2}=\left\{\left(\xi_{1}, \xi_{2}, t\right): \xi_{1} \in S_{1}, \xi_{2} \in S_{2},-1 \leq t \leq 1\right\}$,

- $\Sigma_{3}=\left\{\left(\xi_{1}, \xi_{2}, t\right): \xi_{1} \in S_{1}, \xi_{2} \in S_{3},-1 \leq t \leq 1\right\}$,

- $\Sigma_{4}=\left\{\left(\xi_{1}, \xi_{2}, t\right): \xi_{1} \in S_{2}, \xi_{2} \in S_{1},-1 \leq t \leq 1\right\}$,

- $\Sigma_{5}=\left\{\left(\xi_{1}, \xi_{2}, t\right): \xi_{1} \in S_{2}, \xi_{2} \in S_{2},-1 \leq t \leq 1\right\}$,

- $\Sigma_{6}=\left\{\left(\xi_{1}, \xi_{2}, t\right): \xi_{1} \in S_{2}, \xi_{2} \in S_{3},-1 \leq t \leq 1\right\}$, 
- $\Sigma_{7}=\left\{\left(\xi_{1}, \xi_{2}, t\right): \xi_{1} \in S_{3}, \xi_{2} \in S_{1},-1 \leq t \leq 1\right\}$,

- $\Sigma_{8}=\left\{\left(\xi_{1}, \xi_{2}, t\right): \xi_{1} \in S_{3}, \xi_{2} \in S_{2},-1 \leq t \leq 1\right\}$,

- $\Sigma_{9}=\left\{\left(\xi_{1}, \xi_{2}, t\right): \xi_{1} \in S_{3}, \xi_{2} \in S_{3},-1 \leq t \leq 1\right\}$.

We first prove that $\mathscr{I}_{0}$ covers the subsets $\Sigma_{2}, \Sigma_{4}, \Sigma_{5}, \Sigma_{6}, \Sigma_{8}$. If $\left(\xi_{1}, \xi_{2}, t\right) \in \Sigma_{5}$, then

$$
\left|\xi_{1}\right|^{2}+\left|\xi_{2}\right|^{2} \leq\left(\frac{\sqrt{2}}{2}\right)^{2}+\left(\frac{\sqrt{2}}{2}\right)^{2}=1
$$

so

$$
\left.|| \xi_{1}\right|^{2}+\left|\xi_{2}\right|^{2}+i t \mid \leq \sqrt{1+1}=\sqrt{2}<2
$$

Hence $\Sigma_{5} \subset \mathscr{Y}_{0}$.

If $\left(\xi_{1}, \xi_{2}, t\right) \in \Sigma_{2}$, then

$$
\left|\xi_{1}\right|^{2}+\left|\xi_{2}\right|^{2} \leq 1+\frac{\sqrt{2}}{2}=\frac{3}{2}
$$

so

$$
\left.|| \xi_{1}\right|^{2}+\left|\xi_{2}\right|^{2}+i t \mid \leq \sqrt{\left(\frac{3}{2}\right)^{2}+1}=\sqrt{\frac{13}{4}}<2 .
$$

Therefore $\Sigma_{2} \subset \mathscr{I}_{0}$.

Similarly, we can show that $\Sigma_{4}, \Sigma_{6}, \Sigma_{8}$ are included in $\mathscr{Y}_{0}$.

In order to prove this theorem, it is sufficient to prove that the remaining four subsets are covered by some Heisenberg spheres.

For the set $\Sigma_{9}$, we consider the map $N_{\left((1,1)^{T}, 0\right)} R N_{\left((1,1)^{T}, 0\right)}^{-1}$. The isometric sphere $\mathscr{B}_{1}$ of this map is the Cygan sphere centered at the point $\left((1,1)^{T}, 0,0\right)$ (in horospherical coordinates) with radius 1 . The boundary of $\mathscr{B}_{1}$ is a Heisenberg sphere given by

$$
\mathscr{S}_{1}=\left\{|| \xi_{1}-\left.1\right|^{2}+\left|\xi_{2}-1\right|^{2}+i\left(t+2 \Im\left(\xi_{1}+\xi_{2}\right)\right) \mid=2\right\} .
$$

If $\left(\xi_{1}, \xi_{2}, t\right) \in \Sigma_{9}$, then $\xi_{1} \in S_{3}, \xi_{2} \in S_{3},-1 \leq t \leq 1$. We get that

$$
0 \leq \Im \xi_{i} \leq \frac{1}{2}, \quad\left|\xi_{i}-1\right|^{2} \leq \frac{1}{2},
$$

so

$$
-1 \leq t+2 \Im\left(\xi_{1}+\xi_{2}\right) \leq 3 .
$$

Let $T=N_{\left((0,0)^{T}, 2\right)}$. It is easy to see that the subset

$$
\left\{\left(\xi_{1}, \xi_{2}, t\right): \xi_{1} \in S_{3}, \xi_{2} \in S_{3},-1 \leq t+2 \Im\left(\xi_{1}+\xi_{2}\right) \leq 1\right\}
$$

lies inside $\mathscr{S}_{1}$ and the set

$$
\left\{\left(\xi_{1}, \xi_{2}, t\right): \xi_{1} \in S_{3}, \xi_{2} \in S_{3}, 1 \leq t+2 \Im\left(\xi_{1}+\xi_{2}\right) \leq 3\right\}
$$


lies inside

$$
T^{-1}\left(\mathscr{Y}_{1}\right)=\left\{|| \xi_{1}-\left.1\right|^{2}+\left|\xi_{2}-1\right|^{2}+i\left(t-2+2 \Im\left(\xi_{1}+\xi_{2}\right)\right) \mid=2\right\} .
$$

Therefore, $\mathscr{Y}_{1}$ and $T^{-1}\left(\mathscr{S}_{1}\right)$ cover the set $\Sigma_{9}$.

For the set $\Sigma_{7}$, we consider the map $N_{\left((1, i)^{T}, 0\right)} R N_{\left((1, i)^{T}, 0\right)}^{-1}$. The isometric sphere $\mathscr{B}_{2}$ of this map is the Cygan sphere centered at the point $\left((1, i)^{T}, 0,0\right)$. The boundary of $\mathscr{P}_{2}$ is given by

$$
\mathscr{S}_{2}=\left\{|| \xi_{1}-\left.1\right|^{2}+\left|\xi_{2}-i\right|^{2}+i\left(t+2 \Im\left(\xi_{1}\right)+2 \Re\left(\xi_{2}\right)\right) \mid=2\right\} .
$$

If $\left(\xi_{1}, \xi_{2}, t\right) \in \Sigma_{7}$, then $\xi_{1} \in S_{3}, \xi_{2} \in S_{1},-1 \leq t \leq 1$. We get that

$$
0 \leq \Im \xi_{1} \leq \frac{1}{2}, \quad 0 \leq \Re \xi_{2} \leq \frac{1}{2}, \quad\left|\xi_{1}-1\right|^{2} \leq \frac{1}{2}, \quad\left|\xi_{2}-i\right|^{2} \leq \frac{1}{2},
$$

so

$$
-2 \leq t+2 \Im\left(\xi_{1}+\xi_{2}\right) \leq 2 .
$$

If $-1 \leq t+2 \Im\left(\xi_{1}+\xi_{2}\right) \leq 1$, then the subset

$$
\left\{\left(\xi_{1}, \xi_{2}, t\right): \xi_{1} \in S_{3}, \xi_{2} \in S_{1},-1 \leq t+2 \Im\left(\xi_{1}+\xi_{2}\right) \leq 1\right\}
$$

lies inside $\mathscr{S}_{2}$.

If $-2 \leq t+2 \Im\left(\xi_{1}+\xi_{2}\right) \leq-1$, then the subset

$$
\left\{\left(\xi_{1}, \xi_{2}, t\right): \xi_{1} \in S_{3}, \xi_{2} \in S_{1},-2 \leq t+2 \Im\left(\xi_{1}+\xi_{2}\right) \leq-1\right\}
$$

lies inside $T\left(\mathscr{S}_{2}\right)$.

If $1 \leq t+2 \Im\left(\xi_{1}+\xi_{2}\right) \leq 2$, then the subset

$$
\left\{\left(\xi_{1}, \xi_{2}, t\right): \xi_{1} \in S_{3}, \xi_{2} \in S_{1}, 1 \leq t+2 \Im\left(\xi_{1}+\xi_{2}\right) \leq 2\right\}
$$

lies inside $T^{-1}\left(\mathscr{\varphi}_{2}\right)$.

For the set $\Sigma_{1}$, we consider the map $N_{\left((i, i)^{T}, 0\right)} R N_{\left((i, i)^{T}, 0\right)}^{-1}$. The isometric sphere $\mathscr{B}_{3}$ of this map is the Cygan sphere centered at the point $\left((i, i)^{T}, 0,0\right)$. The boundary of $\mathscr{B}_{3}$ is a Heisenberg sphere given by

$$
\mathscr{S}_{3}=\left\{|| \xi_{1}-\left.1\right|^{2}+\left|\xi_{2}-1\right|^{2}+i\left(t+2 \Im\left(\xi_{1}+\xi_{2}\right)\right) \mid=2\right\} .
$$

If $\left(\xi_{1}, \xi_{2}, t\right) \in \Sigma_{1}$, then $\xi_{1} \in S_{1}, \xi_{2} \in S_{1},-1 \leq t \leq 1$. We get that

$$
0 \leq \Re \xi_{i} \leq \frac{1}{2},\left|\xi_{i}-1\right|^{2} \leq \frac{1}{2}
$$

so

$$
-3 \leq t+2 \Im\left(\xi_{1}+\xi_{2}\right) \leq 1 .
$$

As before, we can see that $\Sigma_{1}$ is covered by the Heisenberg spheres corresponding to the maps

$$
N_{\left((i, i)^{T}, 0\right)} R N_{\left((i, i)^{T}, 0\right)}^{-1} \quad \text { and } \quad T N_{\left((i, i)^{T}, 0\right)} R N_{\left((i, i)^{T}, 0\right)}^{-1} T^{-1} .
$$


It remains to consider the set $\Sigma_{3}$. We consider the map $N_{\left((i, 1)^{T}, 0\right)} R N_{\left((i, 1)^{T}, 0\right)}^{-1}$. The isometric sphere $\mathscr{P}_{4}$ of this map is the Cygan sphere centered at the point $\left((i, 1)^{T}, 0,0\right)$. The boundary of $\mathscr{B}_{4}$ is a Heisenberg sphere given by

$$
\mathscr{S}_{4}=\left\{|| \xi_{1}-\left.i\right|^{2}+\left|\xi_{2}-1\right|^{2}+i\left(t-2 \Re\left(\xi_{1}\right)+2 \mathfrak{\Im}\left(\xi_{2}\right)\right) \mid=2\right\} .
$$

If $\left(\xi_{1}, \xi_{2}, t\right) \in \Sigma_{1}$, then $\xi_{1} \in S_{1}, \xi_{2} \in S_{3},-1 \leq t \leq 1$. We get that

$$
0 \leq \Re \xi_{1} \leq \frac{1}{2}, \quad 0 \leq \Im \xi_{1} \leq \frac{1}{2}, \quad\left|\xi_{1}-i\right|^{2} \leq \frac{1}{2}, \quad\left|\xi_{2}-1\right|^{2} \leq \frac{1}{2},
$$

so

$$
-2 \leq t-2 \Re\left(\xi_{1}\right)+2 \Im\left(\xi_{2}\right) \leq 2 .
$$

If $-1 \leq t-2 \Re\left(\xi_{1}\right)+2 \Im\left(\xi_{2}\right) \leq 1$, then the subset

$$
\left\{\left(\xi_{1}, \xi_{2}, t\right): \xi_{1} \in S_{3}, \xi_{2} \in S_{1},-1 \leq t-2 \Re\left(\xi_{1}\right)+2 \Im\left(\xi_{2}\right) \leq 1\right\}
$$

lies inside $\mathscr{S}_{4}$.

If $-2 \leq t-2 \Re\left(\xi_{1}\right)+2 \Im\left(\xi_{2}\right) \leq-1$, then the subset

$$
\left\{\left(\xi_{1}, \xi_{2}, t\right): \xi_{1} \in S_{3}, \xi_{2} \in S_{1},-2 \leq t-2 \Re\left(\xi_{1}\right)+2 \Im\left(\xi_{2}\right) \leq-1\right\}
$$

lies inside $T\left(\mathscr{Y}_{4}\right)$.

If $1 \leq t-2 \Re\left(\xi_{1}\right)+2 \Im\left(\xi_{2}\right) \leq 2$, then the subset

$$
\left\{\left(\xi_{1}, \xi_{2}, t\right): \xi_{1} \in S_{3}, \xi_{2} \in S_{1}, 1 \leq t-2 \Re\left(\xi_{1}\right)+2 \Im\left(\xi_{2}\right) \leq 2\right\}
$$

lies inside $T^{-1}\left(\mathscr{S}_{4}\right)$. Thus $\Sigma_{3}$ is covered by the Heisenberg spheres corresponding to the maps

$$
N_{\left((i, 1)^{T}, 0\right)} R N_{\left((i, 1)^{T}, 0\right)}^{-1}, \quad T N_{\left((i, 1)^{T}, 0\right)} R N_{\left((i, 1)^{T}, 0\right)}^{-1} T^{-1},
$$

and

$$
T^{-1} N_{\left((i, 1)^{T}, 0\right)} R N_{\left((i, 1)^{T}, 0\right)}^{-1} T .
$$

Remark 9. This method works for the other higher-dimensional Euclidean Picard modular groups $\mathrm{PU}\left(n, 1 ; \mathrm{O}_{d}\right)$ for $d=2,7,11$ and $n \geq 3$. But the calculation will be more complicated. For example, the set $\Sigma$ will be decomposed into smaller parts. Then one needs more Heisenberg spheres to cover the set $\Sigma$ which contains the fundamental set.

\section{Acknowledgements}

We would like to thank D. Allcock for his interest in our work and for explaining to us some results in [Allcock 1999; 2000a; 2000b]. This work was supported by NSF (grant number 11071059) and NSF (grant number 11371126). B. Xie was also supported by NSF (grant number 11201134) and the young teachers support program of Hunan University. 


\section{References}

[Allcock 1999] D. Allcock, "Reflection groups on the octave hyperbolic plane", J. Algebra 213:2 (1999), 467-498. MR 2000e:17028 Zbl 0932.51002

[Allcock 2000a] D. Allcock, "New complex- and quaternion-hyperbolic reflection groups", Duke Math. J. 103:2 (2000), 303-333. MR 2001f:11105 Zbl 0962.22007

[Allcock 2000b] D. Allcock, "The Leech lattice and complex hyperbolic reflections", Invent. Math. 140:2 (2000), 283-301. MR 2002b:11091 Zbl 1012.11053

[Bianchi 1892] L. Bianchi, "Sui gruppi di sostituzioni lineari con coefficienti appartenenti a corpi quadratici immaginarî”, Math. Ann. 40:3 (1892), 332-412. MR 1510727 JFM 24.0188.02

[Cohn 1968] P. M. Cohn, "A presentation of $\mathrm{SL}_{2}$ for Euclidean imaginary quadratic number fields", Mathematika 15 (1968), 156-163. MR 38 \#4568 Zbl 0169.34501

[Falbel and Parker 2006] E. Falbel and J. R. Parker, "The geometry of the Eisenstein-Picard modular group”, Duke Math. J. 131:2 (2006), 249-289. MR 2007f:22011 Zbl 1109.22007

[Falbel et al. 2011a] E. Falbel, G. Francsics, P. D. Lax, and J. R. Parker, "Generators of a Picard modular group in two complex dimensions", Proc. Amer. Math. Soc. 139:7 (2011), 2439-2447. MR 2012d:22014 Zbl 1222.32038

[Falbel et al. 2011b] E. Falbel, G. Francsics, and J. R. Parker, "The geometry of the Gauss-Picard modular group", Math. Ann. 349:2 (2011), 459-508. MR 2011k:22009 Zbl 1213.14049

[Francsics and Lax 2005a] G. Francsics and P. D. Lax, "A semi-explicit fundamental domain for a Picard modular group in complex hyperbolic space", pp. 211-226 in Geometric analysis of PDE and several complex variables, edited by S. Chanillo et al., Contemp. Math. 368, Amer. Math. Soc., Providence, RI, 2005. MR 2006b:22011 Zbl 1065.22007

[Francsics and Lax 2005b] G. Francsics and P. Lax, "An explicit fundamental domain for the Picard modular group in two complex dimensions”, preprint, 2005. arXiv math/0509708

[Francsics and Lax 2006] G. Francsics and P. D. Lax, "Analysis of a Picard modular group", Proc. Natl. Acad. Sci. USA 103:30 (2006), 11103-11105. MR 2007h:11048 Zbl 1206.11048

[Goldman 1999] W. M. Goldman, Complex hyperbolic geometry, Clarendon Press, Oxford, 1999. MR 2000g:32029 Zbl 0939.32024

[Mostow 1980] G. D. Mostow, "On a remarkable class of polyhedra in complex hyperbolic space", Pacific J. Math. 86:1 (1980), 171-276. MR 82a:22011 Zbl 0456.22012

[Parker 2010] J. R. Parker, "Notes on complex hyperbolic geometry", preprint, 2010, Available at http://www.icts.res.in/media/uploads/Program/Files/NCHG.pdf.

[Picard 1883] E. Picard, "Sur des fonctions de deux variables indépendantes analogues aux fonctions modulaires”, Acta Math. 2:1 (1883), 114-135. MR 1554595 JFM 15.0432.01

[Picard 1884] E. Picard, "Sur les formes quadratiques ternaires indéfinies á indéterminées conjuguées et sur les fonctions hyperfuchsiennes correspondantes", Acta Math. 5:1 (1884), 121-182. MR 1554651 JFM 16.0385.01

[Swan 1971] R. G. Swan, "Generators and relations for certain special linear groups", Advances in Math. 6 (1971), 1-77. MR 44 \#1741 Zbl 0221.20060

[Wang et al. 2011] J. Wang, Y. Xiao, and B. Xie, "Generators of the Eisenstein-Picard modular group”, J. Aust. Math. Soc. 91:3 (2011), 421-429. MR 2900617 Zbl 1246.32023

[Xie et al. 2013] B. Xie, J. Wang, and Y. Jiang, "Generators of the Eisenstein-Picard modular group in three complex dimensions”, Glasg. Math. J. 55:3 (2013), 645-654. MR 3084667 Zbl 1273.32027 
[Zhao 2012] T. Zhao, "Generators for the Euclidean Picard modular groups", Trans. Amer. Math. Soc. 364:6 (2012), 3241-3263. MR 2888244 Zbl 1250.22014

Received January 11, 2014. Revised April 27, 2014.

BaoHua Xie

COLlege of MATHEMATICS AND ECONOMETRICS

HUNAN UNIVERSITY

CHANGSHA, 410082

CHINA

xiexbh@hnu.edu.cn

JIEYAN WANG

ACAdEMy of Mathematics and Systems SCIENCE

CHINESE ACADEMY OF SCIENCES

BEIJING, 100190

CHINA

jywang@hnu.edu.cn

YUEPING JIANG

COLlEGE OF MATHEMATICS AND ECONOMETRICS

HUNAN UNIVERSITY

Changsha, 410082

CHINA

ypjiang@hnu.edu.cn 


\title{
PACIFIC JOURNAL OF MATHEMATICS
}

\author{
msp.org/pjm
}

Founded in 1951 by E. F. Beckenbach (1906-1982) and F. Wolf (1904-1989)

\section{EDITORS}

Don Blasius (Managing Editor)

Department of Mathematics

University of California

Los Angeles, CA 90095-1555

blasius@math.ucla.edu

\author{
Paul Balmer \\ Department of Mathematics \\ University of California \\ Los Angeles, CA 90095-1555 \\ balmer@math.ucla.edu \\ Robert Finn \\ Department of Mathematics \\ Stanford University \\ Stanford, CA 94305-2125 \\ finn@math.stanford.edu \\ Sorin Popa \\ Department of Mathematics \\ University of California \\ Los Angeles, CA 90095-1555 \\ popa@math.ucla.edu
}

\author{
Vyjayanthi Chari \\ Department of Mathematics \\ University of California \\ Riverside, CA 92521-0135 \\ chari@math.ucr.edu \\ Kefeng Liu \\ Department of Mathematics \\ University of California \\ Los Angeles, CA 90095-1555 \\ liu@math.ucla.edu \\ Jie Qing \\ Department of Mathematics \\ University of California \\ Santa Cruz, CA 95064 \\ qing@ cats.ucsc.edu
}

\section{PRODUCTION}

Silvio Levy, Scientific Editor, production@msp.org

\section{SUPPORTING INSTITUTIONS}

ACADEMIA SINICA, TAIPEI

CALIFORNIA INST. OF TECHNOLOGY

INST. DE MATEMÁTICA PURA E APLICADA

KEIO UNIVERSITY

MATH. SCIENCES RESEARCH INSTITUTE

NEW MEXICO STATE UNIV.

OREGON STATE UNIV.

\author{
STANFORD UNIVERSITY \\ UNIV. OF BRITISH COLUMBIA \\ UNIV. OF CALIFORNIA, BERKELEY \\ UNIV. OF CALIFORNIA, DAVIS \\ UNIV. OF CALIFORNIA, LOS ANGELES \\ UNIV. OF CALIFORNIA, RIVERSIDE \\ UNIV. OF CALIFORNIA, SAN DIEGO \\ UNIV. OF CALIF., SANTA BARBARA
}

\author{
Daryl Cooper \\ Department of Mathematics \\ University of California \\ Santa Barbara, CA 93106-3080 \\ cooper@math.ucsb.edu \\ Jiang-Hua Lu \\ Department of Mathematics \\ The University of Hong Kong \\ Pokfulam Rd., Hong Kong \\ jhlu@maths.hku.hk \\ Paul Yang \\ Department of Mathematics \\ Princeton University \\ Princeton NJ 08544-1000 \\ yang@math.princeton.edu
}

These supporting institutions contribute to the cost of publication of this Journal, but they are not owners or publishers and have no responsibility for its contents or policies.

See inside back cover or msp.org/pjm for submission instructions.

The subscription price for 2015 is US \$420/year for the electronic version, and \$570/year for print and electronic.

Subscriptions, requests for back issues and changes of subscribers address should be sent to Pacific Journal of Mathematics, P.O. Box 4163, Berkeley, CA 94704-0163, U.S.A. The Pacific Journal of Mathematics is indexed by Mathematical Reviews, Zentralblatt MATH, PASCAL CNRS Index, Referativnyi Zhurnal, Current Mathematical Publications and Web of Knowledge (Science Citation Index).

The Pacific Journal of Mathematics (ISSN 0030-8730) at the University of California, c/o Department of Mathematics, 798 Evans Hall \#3840, Berkeley, CA 94720-3840, is published twelve times a year. Periodical rate postage paid at Berkeley, CA 94704, and additional mailing offices. POSTMASTER: send address changes to Pacific Journal of Mathematics, P.O. Box 4163, Berkeley, CA 94704-0163.

PJM peer review and production are managed by EditFLOW ${ }^{\circledR}$ from Mathematical Sciences Publishers.

\section{PUBLISHED BY}

\section{mathematical sciences publishers \\ nonprofit scientific publishing}

http://msp.org/

(C) 2015 Mathematical Sciences Publishers 


\section{PACIFIC JOURNAL OF MATHEMATICS}

Volume $273 \quad$ No. $1 \quad$ January 2015

Maximal estimates for Schrödinger equations with inverse-square potential 1

ChangXing MiaO, JUNYong Zhang and JiQiang Zheng

Vassiliev Invariants of Virtual Legendrian Knots

PATRICIA CAHN and ASA LEVI

Some results on the generic vanishing of Koszul cohomology via

deformation theory

JIE WANG

Conformal metrics with constant curvature one and finitely many conical

singularities on compact Riemann surfaces

QING Chen, WeI WANG, Yingyi Wu and BIN XU

$\mathbb{Q}$-bases of the Néron-Severi groups of certain elliptic surfaces

MASAMICHI KURODA

On a prime zeta function of a graph

TAKehiro Hasegawa and SEIKEN SAIto

On Whittaker modules for a Lie algebra arising from the 2-dimensional torus 147

SHaObin TAN, QING WANG and ChENGKANG XU

Fréchet quantum supergroups

AXEL DE GOURSAC

Generators of the Gauss-Picard modular group in three complex dimensions

BaoHua Xie, JieYan Wang and YuePing Jiang

Complete characterization of isolated homogeneous hypersurface singularities

STEPHEN YAu and HuAiQING ZuO

A theorem of Mœglin and Waldspurger for covering groups

SHIV PRAKASH PATEL

Spanning trees and random walks on weighted graphs 